\title{
CLASSIFICATION AND SYMMETRIES OF A FAMILY OF CONTINUED FRACTIONS WITH BOUNDED PERIOD LENGTH
}

\author{
K. H. F. CHENG ${ }^{凶}$, R. K. GUY, R. SCHEIDLER and H. C. WILLIAMS
}

(Received 11 February 2011; accepted 1 February 2012)

Communicated by J. O. Shallit

Dedicated to the memory of Alf van der Poorten

\begin{abstract}
It is well known that the regular continued fraction expansion of a quadratic irrational is symmetric about its centre; we refer to this symmetry as horizontal. However, an additional vertical symmetry is exhibited by the continued fraction expansions arising from a family of quadratics known as Schinzel sleepers. This paper provides a method for generating every Schinzel sleeper and investigates their period lengths as well as both their horizontal and vertical symmetries.
\end{abstract}

2010 Mathematics subject classification: primary 11A55; secondary 11Y65.

Keywords and phrases: Schinzel sleeper, continued fraction expansion, period length.

\section{Introduction and notation}

Quadratic irrationals and their regular continued fraction expansions have long been the subject of intense study. These expansions play a significant role in the arithmetic of ideals in a real quadratic order, thus aiding in the computation of the fundamental unit, regulator, and ideal class number of a real quadratic field. Moreover, they have occasionally appeared in cryptographic applications, and their counterparts in function fields play a role in the arithmetic of divisors on real hyperelliptic curves. A vast body of literature exists on this subject; see [4] for the most complete work on this topic to date.

It is well known that the regular continued fraction expansion (henceforth the expansion, for short) of a quadratic irrational $\sqrt{d}$, with $d$ a positive integer that is not a square, is periodic and of the form

$$
\sqrt{d}=\left(a_{0} ; \overline{a_{1}, a_{2}, \ldots, a_{2}, a_{1}, 2 a_{0}}\right),
$$

The first author is supported in part by the Teaching and Research Fund of the HKIEd. The last two authors are supported in part by the NSERC of Canada.

(C) 2013 Australian Mathematical Publishing Association Inc. 1446-7887/2013 \$16.00 
TABLE 1. Expansions of $\sqrt{30^{2} X^{2}+2 \times 180 X+180}$ for $17 \leq X \leq 21$.

\begin{tabular}{cc}
\hline$\sqrt{266400}$ & $(516 ; \overline{7,5,1,27,1,5,7,1032})$ \\
$\sqrt{298260}$ & $(546 ; \overline{7,1,1,2,2,7,5,1,29,1,1,67,1,3,7,2,1,120,}$ \\
$\sqrt{331920}$ & $\frac{(576 ; \overline{8,1152})}{1,2,7,3,1,67,1,1,29,1,5,7,2,2,1,1,7,1092)}$ \\
$\sqrt{367380}$ & $(606 ; \overline{8,2,2,1,1,7,1,5,33,1,1,75,3,1,7,1,2,134}$ \\
$\sqrt{404640}$ & $\left.\frac{(636 ; \overline{8,1,5,35,5,1,8,1272})}{2,1,7,1,3,75,1,1,33,5,1,7,1,1,2,2,1212}\right)$ \\
\hline
\end{tabular}

which is symmetric, apart from the last partial quotient. In general, the length of the expansion (1.1) is of order $\sqrt{d}$. However, there exist parameterized families of continued fractions with bounded period length, which were aptly termed sleepers by Kaplansky [5]. Schinzel [11, 12] completely settled the question of when exactly an integer-valued polynomial $D(X)$ of arbitrary degree is a sleeper.

In addition to their generally exponential length, expansions of quadratic surds tend to be notoriously hard to predict. However, for a certain family of sleepers $D(X)$, the expansion of $\sqrt{D(X)}$ can be explicitly written down. This family is referred to as the Schinzel sleepers and takes the form $D(X)=A^{2} X^{2}+2 B X+C$ with $A \in \mathbb{N}$ and $B, C, X \in \mathbb{Z}$ satisfying the Schinzel condition

$$
B^{2}-A^{2} C \mid 4 \operatorname{gcd}\left(A^{2}, B\right)^{2} .
$$

In [10], Schinzel proved that if $D(X)=A^{2} X^{2}+2 B X+C$ is a quadratic polynomial with integer coefficients, then the period length of the expansion of $\sqrt{D(X)}$ is bounded as $X$ varies if and only if $A, B, C$ satisfy (1.2). An example of a Schinzel sleeper is given by $D(X)=30^{2} X^{2}+2 \times 180 X+180$. The expansions of $\sqrt{D(X)}$ for $X=17,18,19,20,21$ are given in Table 1 .

Cheng and Williams [2] gave an explicit description of the expansion of $\sqrt{D(X)}$ for all sufficiently large integers $X$ when $D(X)$ is a Schinzel sleeper, and found that it is of the form

$$
\left(q_{0}(X) ; \overline{\mathcal{S}_{0}, q_{1}(X), \mathcal{S}_{1}, q_{2}(X), \ldots, \mathcal{S}_{\kappa-1}, q_{\kappa}(X)}\right),
$$

where the period comprises $\kappa$ segments, each consisting of a string $\mathcal{S}_{i}$, possibly empty, but usually an expansion of a rational number, followed by a linear function $q_{i+1}(X)$. Note that comparing (1.3) with (1.1) shows that $q_{0}(X)=a_{0}$ and $q_{\kappa}(X)=2 a_{0}=2 q_{0}(X)$. Using the representation in (1.3), the first line in Table 1 can be rewritten as

$$
\sqrt{266400}=\left(516 ; \overline{\left.\emptyset, 7, \frac{12}{2}, 27, \frac{12}{10}, 7, \emptyset, 1032\right)},\right.
$$


TABLE 2. The expansions from Table 1 in more compact form.

\begin{tabular}{lr}
\hline$\sqrt{266400}$ & $\left(516 ; \overline{\left.\emptyset, 7, \frac{12}{2}, 27, \frac{12}{10}, 7, \emptyset, 1032\right)}\right.$ \\
$\sqrt{298260}$ & $\left(546 ; \frac{}{\emptyset, 7, \frac{12}{7}, 7, \frac{12}{2}, 29, \frac{12}{6}, 67, \frac{12}{9}, 7, \frac{12}{4}, 120,}\right.$ \\
$\sqrt{331920}$ & $\left.\frac{12}{8}, 7, \frac{12}{3}, 67, \frac{12}{6}, 29, \frac{12}{10}, 7, \frac{12}{5}, 7, \emptyset, 1092\right)$ \\
$\sqrt{367380}$ & $\left(606 ; \frac{\left(576 ; \bar{\emptyset}, 8, \emptyset, \frac{12}{5}, 7, \frac{12}{10}, 33, \frac{12}{6}, 75, \frac{12}{3}, 7, \frac{12}{8}, 134,\right.}{\left.\frac{12}{4}, 7, \frac{12}{9}, 75, \frac{12}{6}, 33, \frac{12}{2}, 7, \frac{12}{7}, 8, \emptyset, 1212\right)}\right.$ \\
$\sqrt{404640}$ & $\left(636 ; \emptyset, 8, \frac{12}{10}, 35, \frac{12}{2}, 8, \emptyset, 1272\right)$ \\
\hline
\end{tabular}

where $\mathcal{S}_{0}, \mathcal{S}_{3}$ are empty, and $\mathcal{S}_{1}, \mathcal{S}_{2}$ are given by the even length expansions of $12 / 2$ and $12 / 10$, respectively. All five expansions of Table 1 are rewritten in this fashion in Table 2.

We will see that if $D(X)$ is a Schinzel sleeper, then the expansion of $\sqrt{D(X)}$ not only has the usual horizontal periodicity and symmetry, but also exhibits a vertical periodicity and symmetry as $X$ varies along certain congruence classes.

The authors of [2] also provided an upper bound on the period length of the expansion of $\sqrt{D(X)}$ and, without proof, related the value of $\kappa$ to a rank of apparition in a certain Lucas sequence. We will investigate this connection in more detail in Section 2 and focus on specific values of $\kappa$ in Section 3. The continued fraction expansion is explicitly described in Section 4, and the aforementioned vertical symmetry will be explored in Section 5 . We conclude with a systematic method for characterizing and explicitly generating all Schinzel sleepers in Section 6.

The notation used throughout this paper is summarized in Table 3 and is consistent with [2]. In particular, $D(X)=A^{2} X^{2}+2 B X+C$ will always represent a Schinzel sleeper.

Note that $D(X)=(A X+B / A)^{2}-\Delta / A^{2}$ with $\Delta=B^{2}-A^{2} C$. We assume that $\Delta \neq 0$, as otherwise $D(X)$ is an integer square for all $X$. The condition in Table 3 that $X$ be sufficiently large is to ensure that the expansion of $\sqrt{D(X)}$ begins with the term $A X+\lfloor B / A\rfloor-\eta$; see [2, Theorem 2.1] for an explicit lower bound on $X$.

With the notation of Table 3 , we have $B^{2}-A^{2} C=\sigma \Delta_{1} \Delta_{2}^{2} \Delta_{4}^{4}$. Since $\Delta_{1}$ is squarefree, it is easy to see that $G=\operatorname{gcd}(A, B)=\operatorname{gcd}\left(A, \Delta_{2} \Delta_{4}^{2}\right)$, so that $\operatorname{gcd}\left(A^{\prime}, \Delta^{\prime}\right)=1$. Now (1.2) implies that $\Delta_{1} \Delta_{2} \Delta_{4}^{2} \mid 2 \operatorname{gcd}\left(A^{2}, B\right)$, so that $\Delta_{1} \Delta^{\prime} \mid 2 \operatorname{gcd}\left(G A^{\prime 2}, B^{\prime}\right)$. Since $A^{\prime}$ and $B^{\prime}$ are coprime, the Schinzel condition (1.2) is thus equivalent to

$$
\Delta_{1} \Delta^{\prime} \mid 2 \operatorname{gcd}\left(G, B^{\prime}\right) .
$$

The quantity $\Delta^{\prime}$ will turn out to be crucial in determining the period length and analysing the symmetry properties of the expansion of $\sqrt{D(X)}$. 
TABLE 3. Notation used throughout this paper.

\begin{tabular}{ll}
\hline Symbol & Meaning \\
\hline$A, B, C$ & Integers with $A>0$ that satisfy $(1.2)$ \\
$X$ & Sufficiently large integer \\
$D(X)$ & $D(X)=A^{2} X^{2}+2 B X+C$ is a Schinzel sleeper \\
$\kappa$ & Number of segments in the continued fraction expansion of $\sqrt{D(X)}$ \\
$\Delta$ & $\Delta=B^{2}-A^{2} C$ \\
$\sigma$ & $\sigma=\operatorname{sgn}(\Delta)$ \\
$r$ & $r \equiv B$ mod $A$ with $0 \leq r<A$ \\
$\eta$ & $\eta=1$ if $A \mid B$ and $\sigma=1 ;$ otherwise $\eta=0$ \\
$\Delta_{1}, \Delta_{2}, \Delta_{4}$ & $|\Delta|=\Delta_{1} \Delta_{2}^{2} \Delta_{4}^{4}$ with $\Delta_{1}, \Delta_{2}$ square-free \\
$G$ & $G=\operatorname{gcd}(A, B)=\operatorname{gcd}\left(A, \Delta_{2} \Delta_{4}^{2}\right)$ \\
$A^{\prime}, B^{\prime}$ & $A^{\prime}=A / G, B^{\prime}=B / G$ \\
$\Delta^{\prime}$ & $\Delta^{\prime}=\Delta_{2} \Delta_{4}^{2} / G$ \\
$\tau$ & $\tau=1$ if $\Delta_{1} \Delta^{\prime}$ is odd, $\tau=2$ if $\Delta_{1} \Delta^{\prime}$ is even \\
$K$ & $X \equiv K$ mod $\Delta^{\prime}$ with $0 \leq K<\Delta^{\prime}$ \\
$T$ & $T=2\left(A^{2} K+B\right) / \Delta_{2} \Delta_{4}^{2}$ \\
$P$ & $P=T^{2} / \Delta_{1}-2 \sigma$ \\
$\delta$ & $\delta=\operatorname{gcd}\left(\Delta^{\prime}, T / \Delta_{1}\right)$ \\
$\alpha, \beta$ & Roots of $x^{2}-P x+1=0$ \\
$U_{i}$ & Lucas sequence arising from $\alpha, \beta$ as defined in $(2.1)$ \\
$Z_{i}$ & Auxiliary sequence defined in $(2.3)$ \\
$\varepsilon_{i}$ & Parity of $i \in \mathbb{Z}$, that is, $\varepsilon_{i}=\left(1-(-1)^{i}\right) / 2$ \\
$\omega(m)$ & Rank of apparition of $m$ in the Lucas sequence $\left\{U_{i}\right\}$ \\
$d_{i}$ & $d_{0}=\Delta^{\prime}$ and $d_{i}$ defined in $(4.1)$ for $1 \leq i \leq \kappa$ \\
$g_{i}$ & Defined in $(4.2)$ for $1 \leq i \leq \kappa$ \\
$r_{i}$ & $r_{0}=(r+A \eta) / G$ and $r_{i}$ defined in $(4.3)$ for $1 \leq i \leq \kappa$ \\
$q_{i}(X)$ & $q_{0}(X)=A X+\lfloor B / A\rfloor-\eta$ and $q_{i}(X)$ defined in $(4.4)$ for $1 \leq i \leq \kappa$ \\
$\mathcal{S}_{i}$ & String of partial quotients in the expansion of $A^{\prime} \Delta^{\prime} / d_{i} r_{i} ;$ \\
& see Theorem 4.1 \\
\hline &
\end{tabular}

\section{Number of segments in the expansion of $\sqrt{D(X)}$}

We now investigate the connection between $\kappa$ as given in (1.3) and a rank of apparition in a certain Lucas sequence in more detail. Note first that with the notation of Table 3,

$$
\frac{T}{\Delta_{1}}=\frac{2 G A^{\prime 2} K+2 B^{\prime}}{\Delta_{1} \Delta^{\prime}},
$$

so (1.4) immediately implies that $T / \Delta_{1}$ is an integer, and hence so are $P$ and $\delta$. 
Let $\alpha$ and $\beta$ be the roots of $x^{2}-P x+1=0$. Then $\alpha+\beta=P$ and $\alpha \beta=1$. The corresponding Lucas sequence is given by

$$
U_{i}= \begin{cases}\frac{\alpha^{i}-\beta^{i}}{\alpha-\beta} & \text { if } \alpha \neq \beta, \\ i \alpha^{i-1} & \text { if } \alpha=\beta,\end{cases}
$$

when $i \geq 0$. Then $U_{0}=0, U_{1}=1, U_{2}=P$ and $U_{i+1}=P U_{i}-U_{i-1}$ for $i \in \mathbb{N}$. It is well known (see, for example, Williams [16, display (4.2.27), p. 76], or one may verify it directly using (2.1)), that $U_{i+j}=U_{i+1} U_{j}-U_{i} U_{j-1}$ when $i \geq 0$ and $j \geq 1$. In particular, if $j=i+1$, we obtain

$$
U_{2 i+1}=U_{i+1}^{2}-U_{i}^{2}=\left(U_{i+1}-\sigma U_{i}\right)\left(U_{i+1}+\sigma U_{i}\right)
$$

for all $i \geq 0$, where $\sigma=\operatorname{sgn}(\Delta)$. Our characterization of $\kappa$ requires an auxiliary sequence $\left\{Z_{i}\right\}$. Define

$$
Z_{0}=0, \quad Z_{1}=1, \quad Z_{i+1}=\frac{T}{\Delta_{1}^{\varepsilon_{i}}} Z_{i}-\sigma Z_{i-1},
$$

for all $i \in \mathbb{N}$, where $\varepsilon_{i}=\left(1-(-1)^{i}\right) / 2 \in\{0,1\}$ is the parity of $i \in \mathbb{Z}$. Note that both $\left\{U_{i}\right\}$ and $\left\{Z_{i}\right\}$ are divisibility sequences, that is, the $i$ th term divides the $(i j)$ th term for all $i, j \in \mathbb{N}$; for Lucas sequences, this fact is well known.

We have

$$
Z_{i+3}=P Z_{i+1}-Z_{i-1}
$$

for all $i \in \mathbb{N}$. It is now straightforward to prove that

$$
Z_{2 i}=\frac{T}{\Delta_{1}} U_{i}, \quad Z_{2 i+1}=U_{i+1}+\sigma U_{i},
$$

for all $i \geq 0$, with $U_{i}$ as defined in (2.1). Induction readily yields

$$
\operatorname{gcd}\left(Z_{i}, Z_{i-1}\right)=\operatorname{gcd}\left(U_{i}, U_{i-1}\right)=1
$$

for all $i \in \mathbb{N}$. The following characterization of $\kappa$ is crucial to the results in this section as well as Section 5.

THEOREM 2.1. The number $\mathrm{k}$ of segments in (1.3) is the least positive integer $k$ such that $\Delta^{\prime} \mid Z_{k}$ and $\Delta_{1}^{\varepsilon_{k}}=1$, where $\varepsilon_{k}=\left(1-(-1)^{k}\right) / 2$ is the parity of $k$.

In order to avoid introducing a large amount of currently unnecessary notation, we postpone the proof of this theorem. The result will follow from Theorem 4.1 and Lemma 4.2 below. Note that by Theorem 2.1, $\kappa$ must be even if $\Delta_{1}>1$; in other words, if $\kappa$ is odd, then $|\Delta|$ is a square.

The rank of apparition (if it exists) of $m \in \mathbb{N}$ in an integer sequence $\left\{s_{i}\right\}$ is the least positive integer $k$ such that $m \mid s_{k}$. (The term 'rank of apparition' is a translation 
TABLE 4. $\kappa$ and $\omega\left(\Delta^{\prime} / \delta\right)$ for $\sqrt{30^{2} X^{2}+2 \times 180 X+180}$ with $X \equiv K \bmod 12$.

\begin{tabular}{lcccccccccccc}
\hline$K$ & 0 & 1 & 2 & 3 & 4 & 5 & 6 & 7 & 8 & 9 & 10 & 11 \\
\hline$\kappa$ & 12 & 4 & 12 & 4 & 6 & 4 & 12 & 2 & 12 & 4 & 6 & 4 \\
$\omega\left(\Delta^{\prime} / \delta\right)$ & 6 & 2 & 6 & 2 & 3 & 2 & 6 & 1 & 6 & 2 & 3 & 2 \\
\hline
\end{tabular}

from the French. Ribenboim [9, p. 51] proposed to use the arguably more appropriate wording 'rank of appearance' instead. However, since 'rank of apparition' seems to be widely used throughout the literature, we opted to use this term.) Theorem 2.1 immediately yields the following result.

Corollary 2.2. $\kappa$ is the rank of apparition of $\Delta^{\prime}$ in the sequence $\left\{Z_{i}\right\}$.

For all $m \in \mathbb{N}$, the rank of apparition of $m$ in the Lucas sequence $\left\{U_{i}\right\}$ exists; see $[16$, p. 86]. Henceforth, denote this quantity by $\omega(m)$. Theorem 4.2 in [2] was stated without proof and characterized $\kappa$ as follows.

THEOREM 2.3. If $\kappa$ is odd, then $\kappa=\omega\left(\Delta^{\prime}\right)$, and if $\kappa$ is even, then $\kappa=2 \omega\left(\Delta^{\prime} / \delta\right)$, where $\delta=\operatorname{gcd}\left(\Delta^{\prime}, T / \Delta_{1}\right)$.

Proof. Suppose first that $\kappa$ is odd. For brevity, set $\omega=\omega\left(\Delta^{\prime}\right)$. By Theorem 2.1, $\Delta^{\prime} \mid Z_{\kappa}$. Now

$$
Z_{\kappa}=U_{(\kappa+1) / 2}+\sigma U_{(\kappa-1) / 2}
$$

by (2.4) with $i=(\kappa-1) / 2$, so $Z_{\kappa} \mid U_{\kappa}$ by (2.2). Therefore $\omega \mid \kappa$. Furthermore, $\Delta^{\prime} \mid U_{\omega}$, and $U_{\omega} \mid Z_{2 \omega}$ by (2.4) with $i=\omega$. Thus, $\kappa \leq 2 \omega$ by Theorem 2.1. It follows that $\kappa / \omega \leq 2$. Since $\kappa$ is odd, this forces $\kappa=\omega$.

Now suppose that $\kappa$ is even. For brevity, set $\omega=\omega\left(\Delta^{\prime} / \delta\right)$. By Theorem 2.1, $\Delta^{\prime} \mid Z_{\kappa}$, so (2.4) with $i=\kappa / 2$ implies that $\Delta^{\prime} \mid\left(T / \Delta_{1}\right) U_{\kappa / 2}$. Since $\delta=\operatorname{gcd}\left(\Delta^{\prime}, T / \Delta_{1}\right)$, this implies that $\left(\Delta^{\prime} / \delta\right) \mid U_{\kappa / 2}$, which in turn implies that $\omega \mid(\kappa / 2)$ (see, for example, [16, Theorem 4.3.4, p. 87]), and hence $2 \omega \leq \kappa$.

On the other hand, by (2.4) with $i=\omega$, we see that $Z_{2 \omega}=\left(T / \Delta_{1}\right) U_{\omega}$. Since $\left(\Delta^{\prime} / \delta\right) \mid U_{\omega}$ and $\delta \mid\left(T / \Delta_{1}\right)$, this yields $\Delta^{\prime} \mid Z_{2 \omega}$. By Theorem 2.1, $\kappa \leq 2 \omega$. Therefore $\kappa=2 \omega$.

We illustrate Theorem 2.3 with two examples. The first is a Schinzel sleeper such that $\Delta<0$ and $|\Delta|$ is a square.

Example 2.4. Consider $D(X)=30^{2} X^{2}+2 \times 180 X+180$. Here, $G=A=30, \Delta=$ $-2^{6} \times 3^{4} \times 5^{2}, \Delta_{1}=1, \Delta_{2}=10, \Delta_{4}=6$ and $\Delta^{\prime}=12$. The results for sufficiently large $X$ such that $X \equiv K \bmod 12$, with $0 \leq K \leq 11$, are presented in Table 4 .

For our second example, we have $\Delta>0$ and not a square. 
TABLE 5. $\kappa$ and $\omega\left(\Delta^{\prime} / \delta\right)$ for $\sqrt{119^{2} X^{2}+2 \times 1666 X+98}$ with $X \equiv K \bmod 7$.

\begin{tabular}{lccccccc}
\hline$K$ & 0 & 1 & 2 & 3 & 4 & 5 & 6 \\
\hline$\kappa$ & 6 & 6 & 4 & 14 & 2 & 14 & 4 \\
$\omega\left(\Delta^{\prime} / \delta\right)$ & 3 & 3 & 2 & 7 & 1 & 7 & 2 \\
\hline
\end{tabular}

Example 2.5. Consider $D(X)=119^{2} X^{2}+2 \times 1666 X+98$. Here, $G=A=119, \Delta=$ $2 \times 7^{4} \times 17^{2}, \Delta_{1}=2, \Delta_{2}=17, \Delta_{4}=7$ and $\Delta^{\prime}=7$. For $X \equiv K \bmod 7$ sufficiently large, with $0 \leq K \leq 6$, we present the results in Table 5 .

We conclude this section with an upper bound on $\kappa$; in fact, we provide an upper bound on the rank of apparition in $\left\{U_{i}\right\}$. Using the notation of [16, Section 4.3], set

$$
\epsilon(2)= \begin{cases}0 & \text { if } P \text { is even, } \\ -1 & \text { if } P \text { is odd }\end{cases}
$$

For an odd prime $p$, set $\epsilon(p)=\left(\left(P^{2}-4\right) / p\right)$, the Legendre symbol of $P^{2}-4$ with respect to $p$; note that $P^{2}-4$ is the discriminant of the polynomial $x^{2}-P x+1$ whose zeros define the Lucas sequence $\left\{U_{i}\right\}$.

Let $n \in \mathbb{N}$. Define $\Lambda^{\prime}\left(2^{n}\right)=2^{n-1}(2-\epsilon(2))$ and

$$
\Lambda^{\prime}\left(p^{n}\right)= \begin{cases}p^{n} & \text { if } p \mid P^{2}-4, \\ p^{n-1}(p-\epsilon(p)) / 2 & \text { if } p \nmid P^{2}-4,\end{cases}
$$

for every odd prime $p$. Finally, set $\Lambda^{\prime}(1)=1$, and for all $m \geq 2$ with prime factorization $m=p_{1}^{n_{1}} p_{2}^{n_{2}} \times p_{r}^{n_{r}}$, define $\Lambda^{\prime}(m)$ to be the least common multiple of the $\Lambda^{\prime}\left(p_{i}^{n_{i}}\right)$ for $1 \leq i \leq r$. Note that $\Lambda^{\prime}$ is akin to the Carmichael lambda function, but the above definition is different from [16, Definition 4.3.7, p. 88]. We state a slightly stronger version of the law of apparition given in [16, Theorem 4.3.8, p. 88].

Lemma 2.6. $\omega(m) \mid \Lambda^{\prime}(m)$ for all $m \in \mathbb{N}$.

Proof. It suffices to prove that $m \mid U_{\Lambda^{\prime}(m)}$. For $m=1$, this is obvious. Moreover, $p \mid U_{\Lambda^{\prime}(p)}$ for every prime $p$ : for $p=2$ or $p \mid P^{2}-4$, this is stated in [16, (4.3.1) and (4.3.3), pp. 83-84], respectively; for all other primes $p$, this follows from [16, Theorem 4.3 .1$, p. 85]. By [16, Theorem 4.3.2, p. 85], $p^{n} \mid U_{\Lambda^{\prime}\left(p^{n}\right)}$ for all $n \in \mathbb{N}$.

Let $p$ be a prime divisor of $m$ and write $m=p^{n} m^{\prime}$ with $p \nmid m^{\prime}$. The definition of $U_{i}$ easily yields $U_{i} \mid U_{k i}$ for all $i, k \in \mathbb{N}$, so $p^{n} \mid U_{\Lambda^{\prime}(m)}$, and hence $m \mid U_{\Lambda^{\prime}(m)}$.

Corollary 2.7. We have the following estimate for $\omega$ :

$$
\omega(m) \leq \begin{cases}3 m / 2 & \text { if } m \text { is even and } P \text { is odd }, \\ m & \text { otherwise },\end{cases}
$$

for all $m \in \mathbb{N}$. 
TABLE 6. Upper bounds on $\kappa$.

\begin{tabular}{lcccc}
\hline$\delta$ & 1 & $\geq 2$ & 1 & $\geq 2$ \\
\hline$\left(\Delta^{\prime}, P\right)$ & $($ even, odd) & not (even, odd) \\
Bound on $\kappa$ & $3 \Delta^{\prime}$ & $3 \Delta^{\prime} / 2$ & $2 \Delta^{\prime}$ & $\Delta^{\prime}$ \\
\hline
\end{tabular}

TABLE 7. Upper bounds on $\kappa$ for $\kappa$ even.

\begin{tabular}{lcc}
\hline$\left(\Delta^{\prime} / \delta, P\right)$ & $($ even, odd $)$ & not (even, odd $)$ \\
Bound on $\kappa$ & $3 \Delta^{\prime} / \delta$ & $2 \Delta^{\prime} / \delta$ \\
\hline
\end{tabular}

Proof. This follows from the fact that $\Lambda^{\prime}\left(p^{n}\right) \leq p^{n}$ for every odd prime $p, \Lambda^{\prime}\left(2^{n}\right)=2^{n}$ for $P$ even, and $\Lambda^{\prime}\left(2^{n}\right)=3 \times 2^{n-1}$ for $P$ odd.

Theorem 2.3 now immediately yields the bounds on $\kappa$ in Table 6 .

Note that this slightly strengthens [2, Theorem 4.1] and [1, Theorem 5.2.3]. If $\kappa$ is even, which is always the case when $\Delta_{1}>1$, then the bounds on $\kappa$ can be tightened as in Table 7.

We remark that these bounds are sharp.

Example 2.8. Consider the Schinzel sleeper $D(X)=30^{2} X^{2}+2 \times 180 X+180$ from Example 2.4. For $K=1$, we have $\Delta^{\prime} / \delta=2$ (even), $P=38$ (even), and $\kappa=2 \Delta^{\prime} / \delta=4$. For $K=7$, we obtain $\Delta^{\prime} / \delta=1$ odd and $\kappa=2 \Delta^{\prime} / \delta=2$.

Example 2.9. Consider the Schinzel sleeper $D(X)=119^{2} X^{2}+2 \times 1666 X+168$, with $\Delta_{1}=7, \Delta_{2}=238, \Delta_{4}=1$, and $\Delta^{\prime}=2$. For $K=1$, we have $T / \Delta_{1}=19$, so $\delta=1$ and $\Delta^{\prime} / \delta=2$ is even. Furthermore, $P=2525$ is odd. By Corollary 3.3 below, $\kappa=6=$ $3 \Delta^{\prime} / \delta$.

\section{Specific cases}

Unfortunately, it seems difficult to provide a more practical characterization of $\kappa$ than the one given in Theorem 2.3. This would amount to an a priori determination of the rank of apparition of $\Delta^{\prime}$ or $\Delta^{\prime} / \delta$ in the Lucas sequence $\left\{U_{i}\right\}$, which is generally a challenging task. However, it is possible to characterize small values of $\kappa$ completely and consequently determine $\kappa$ for certain values of $\Delta^{\prime}$. We recall that $P=T^{2} / \Delta_{1}-2 \sigma$.

PROPOSITION 3.1. We have the following characterizations of values of $\kappa$.

$\kappa=1$ if and only if $\Delta_{1}=\Delta^{\prime}=1$.

If $\Delta^{\prime}>1$, then the following hold:

$\kappa=2$ if and only if $\delta=\Delta^{\prime}$ if and only if $P \equiv-2 \sigma \bmod \Delta^{\prime 2}$;

$\kappa=3$ if and only if $P \equiv-\sigma \bmod \Delta^{\prime}$ and $\Delta_{1}=1$; 
$\kappa=4$ if and only if $P \equiv 0 \bmod \Delta^{\prime} / \delta$ and $\delta<\Delta^{\prime}$;

$\kappa=5$ if and only if $P^{2}+\sigma P-1 \equiv 0 \bmod \Delta^{\prime}$ and $\Delta_{1}=1$;

$\kappa=6$ if and only if $P^{2} \equiv 1 \bmod \Delta / \delta, \delta<\Delta^{\prime}$, and $\kappa \neq 3$.

Proof. We compute the first few values of $Z_{i}$ for $i \in \mathbb{N}$ to obtain

$$
\begin{aligned}
Z_{1}=1, & Z_{2}=T / \Delta_{1}, & Z_{3}=P+\sigma, \\
Z_{4}=P Z_{2}, & Z_{5}=P^{2}+\sigma P-1, & Z_{6}=\left(P^{2}-1\right) Z_{2} .
\end{aligned}
$$

It is obvious from Theorem 2.1 that $\kappa=1$ if and only if $\Delta_{1}=\Delta^{\prime}=1$. So assume now that $\Delta^{\prime}>1$. We use Theorem 2.1 as well as (2.5).

Clearly, $\kappa=2$ if and only if $\Delta^{\prime} \mid Z_{2}$, or equivalently, $\delta=\Delta^{\prime}$. Since $\Delta_{1}$ is square-free, $\Delta^{\prime}$ divides $T / \Delta_{1}$ if and only if $\Delta^{\prime 2}$ divides $T^{2} / \Delta_{1}=P+2 \sigma$.

The result for $\kappa=3$ is obvious. Also, $\kappa=4$ if and only if $\kappa \neq 2$ and $\Delta^{\prime} \mid Z_{4}$, which in turn holds if and only if $\delta<\Delta^{\prime}$ and $\left(\Delta^{\prime} / \delta\right) \mid P$.

Next, $\kappa=5$ if and only if $\Delta_{1}=1, \Delta^{\prime} \mid Z_{5}$, and $\Delta^{\prime} \nmid Z_{3} Z_{2}$. But if $\Delta^{\prime} \mid Z_{3}$ or $\Delta^{\prime} \mid Z_{2}$, then $Z_{5} \equiv 0 \bmod \Delta^{\prime}$.

Finally, $\kappa=6$ if and only if $\kappa \neq 2,3, \Delta^{\prime} \mid Z_{6}$ and $\Delta^{\prime} \nmid Z_{4}$. Now again $\kappa \neq 2$ and $\Delta^{\prime} \mid Z_{6}$ if and only if $\delta<\Delta^{\prime}$ and $P^{2} \equiv 1 \bmod \Delta^{\prime} / \delta$. Note that this implies that $\operatorname{gcd}\left(\Delta^{\prime} / \delta, P\right)=1$ and hence $\operatorname{gcd}\left(\Delta^{\prime} / \delta, Z_{4}\right)=1$, so $\Delta^{\prime} \nmid Z_{4}$.

It is now easy to deduce all the $\kappa$ values for certain $\Delta^{\prime}$.

COROLlaRY 3.2. The possible values for $\kappa$ for $\Delta^{\prime}=1$ are as follows:

$$
\begin{aligned}
& \kappa=1 \text { if } \Delta_{1}=1 ; \\
& \kappa=2 \text { if } \Delta_{1}>1 .
\end{aligned}
$$

Corollary 3.3. The possible values for $\kappa$ for $\Delta^{\prime}=2$ are as follows:

$$
\begin{aligned}
& \kappa=2 \text { if } P \equiv 2 \bmod 4 ; \\
& \kappa=3 \text { if } P \text { is odd and } \Delta_{1}=1 ; \\
& \kappa=4 \text { if } P \equiv 0 \text { mod } 4 ; \\
& \kappa=6 \text { if } P \text { is odd and } \Delta_{1}>1 .
\end{aligned}
$$

We remark that the results of van der Poorten and Williams [14] assumed that $\operatorname{gcd}\left(A^{2}, 2 B, C\right)$ is square-free, which in fact forces $\Delta^{\prime} \mid 2$; see Corollary 6.2 below.

PRoposition 3.4. The possible values for $\kappa$ for $\Delta^{\prime}=3$ are as follows:

$$
\begin{aligned}
& \kappa=2 \text { if } P \equiv-2 \sigma \bmod 9 ; \\
& \kappa=3 \text { if } P \equiv-\sigma \bmod 3 \text { and } \Delta_{1}=1 ; \\
& \kappa=4 \text { if } P \equiv 0 \bmod 3 ; \\
& \kappa=6 \text { if } P \equiv-\sigma \bmod 3 \text { and } \Delta_{1}>1 \text {; or if } P \equiv \sigma \text { or } 4 \sigma \bmod 9 .
\end{aligned}
$$

We also consider a few higher powers of 2 .

Proposition 3.5. The possible values for $\kappa$ for $\Delta^{\prime}=4$ are as follows:

$$
\begin{aligned}
& \kappa=2 \text { if } P \equiv-2 \sigma \bmod 16 ; \\
& \kappa=3 \text { if } P \equiv-\sigma \bmod 4 \text { and } \Delta_{1}=1 ;
\end{aligned}
$$


$\kappa=4$ if $P \equiv 2 \bmod 4$ and $P \not \equiv-2 \sigma \bmod 16$;

$\kappa=6$ if $P$ is odd except when $P \equiv-\sigma \bmod 4$ and $\Delta_{1}=1$;

$\kappa=8$ if $P \equiv 0 \bmod 4$.

By computing $Z_{i}$ for $i=8,10,12,14,16$, and noting that $Z_{i}$ is odd for $i$ odd and $P$ even, we obtain the next result.

Proposition 3.6. The possible values for $\kappa$ for $\Delta^{\prime}=8$ are as follows:

$\kappa=2$ if $P \equiv-2 \sigma \bmod 64$

$\kappa=3$ if $P \equiv-\sigma \bmod 8$ and $\Delta_{1}=1$;

$\kappa=4$ if $P \equiv 2 \bmod 4$ and $P \not \equiv-2 \sigma \bmod 8$;

$\kappa=6$ if $P$ is odd except when $P \equiv-\sigma \bmod 8$ and $\Delta_{1}=1$;

$\kappa=8$ if $P \equiv-2 \sigma \bmod 8$ and $P \not \equiv-2 \sigma \bmod 16$;

$\kappa=16$ if $P \equiv 0 \bmod 4$.

It is also straightforward to analyse the cases when $\Delta^{\prime}>3$ is prime and $P \equiv 0, \pm 1$ or $\pm 2 \bmod \Delta^{\prime}$.

Proposition 3.7. Let $\Delta^{\prime}>3$ be prime and $P \equiv 0,1$ or $-1 \bmod \Delta^{\prime}$. Then the following hold:

$\kappa=3$ if and only if $P \equiv-\sigma \bmod \Delta^{\prime}$ and $\Delta_{1}=1$;

$\kappa=4$ if and only if $P \equiv 0 \bmod \Delta^{\prime}$;

$\kappa=6$ if and only if $P \equiv \sigma \bmod \Delta^{\prime}$, or if $P \equiv-\sigma \bmod \Delta^{\prime}$ and $\Delta_{1}>1$.

The case $P \equiv \pm 2 \bmod \Delta^{\prime}$ uses a very different proof technique.

Proposition 3.8. Let $\Delta^{\prime}$ be an odd prime with $P \equiv \pm 2 \bmod \Delta^{\prime}$. Then $\kappa=2$ if and only if $P \equiv-2 \sigma \bmod \Delta^{\prime 2}$, else $\kappa=\Delta^{\prime}$ or $2 \Delta^{\prime}$.

Proof. Since $\Delta^{\prime} \nmid U_{1}=1$, we have $\omega\left(\Delta^{\prime}\right)>1$. Now $\Lambda^{\prime}\left(\Delta^{\prime}\right)=\Delta^{\prime}$, so by Lemma 2.6, $\omega\left(\Delta^{\prime}\right) \mid \Delta^{\prime}$. It follows that $\omega\left(\Delta^{\prime}\right)=\Delta^{\prime}$.

The claim for $\kappa=2$ follows immediately from Proposition 3.1, so suppose that $P \not \equiv-2 \sigma \bmod \Delta^{\prime 2}$. Then $\Delta^{\prime} \nmid Z_{2}$, so $\delta=\operatorname{gcd}\left(\Delta^{\prime}, Z_{2}\right)=1$, and hence $\Delta^{\prime} / \delta=\Delta^{\prime}$. By Theorem 2.3, $\kappa \in\left\{\omega\left(\Delta^{\prime}\right), 2 \omega\left(\Delta^{\prime}\right)\right\}=\left\{\Delta^{\prime}, 2 \Delta^{\prime}\right\}$.

Example 3.9. Recall the sleeper $D(X)=119^{2} X^{2}+2 \times 1666 X+98$ from Example 2.5. We have $\Delta_{1}=2, \sigma=1$ and $\Delta^{\prime}=7$. It is easy to verify that $\delta=1$ for $K=0,1,2,6$. Table 8 lists for $0 \leq K \leq 6$ the corresponding values of $\kappa$ and the relevant congruence conditions on $P$ to apply Propositions 3.1, 3.7 or 3.8.

\section{Continued fraction expansion of $\sqrt{D(X)}$}

We recall the main result (namely Theorem 3.1) of [2] which describes in detail the expansion of $\sqrt{D(X)}$, with $D(X)$ a Schinzel sleeper. In order to obtain the quantities $\mathcal{S}_{i}$ and $q_{i}(X)$ of (1.3), we require some additional notation. 
TABLE 8. Obtaining $\kappa$ for $\sqrt{119^{2} X^{2}+2 \times 1666 X+98}$ from Propositions 3.1, 3.7, 3.8.

\begin{tabular}{cccccc}
\hline$K$ & $\kappa$ & $P \bmod 7$ & $P^{2} \bmod 7$ & $P \bmod 49$ & $\kappa$ obtainable via Proposition(s) \\
\hline 0 & 6 & -1 & 1 & & 3.1 or 3.7 \\
1 & 6 & -1 & 1 & & 3.1 or 3.7 \\
2 & 4 & 0 & & & 3.1 or 3.7 \\
3 & 14 & 2 & & 16 & 3.8 \\
4 & 2 & -2 & & -2 & $3.1,3.7$, or 3.8 \\
5 & 14 & 2 & 9 & 3.8 \\
6 & 4 & 0 & & & or 3.7 \\
\hline
\end{tabular}

Set $d_{0}=\Delta^{\prime}, r_{0}=(r+A \eta) / G$, and for $1 \leq i \leq \kappa$, define $d_{i}, g_{i}, r_{i}$ cyclically by

$$
\begin{gathered}
d_{i}=\operatorname{gcd}\left(\frac{\Delta^{\prime}}{d_{i-1}}, r_{i-1}\right), \\
g_{i}= \begin{cases}0 & \text { if } r_{i-1}=0, \\
d_{i} & \text { if } r_{i-1}=A^{\prime} \Delta^{\prime} / d_{i}, \\
h & \text { if } 0<r_{i-1}<A^{\prime} \Delta^{\prime} / d_{i},\end{cases}
\end{gathered}
$$

where $h r_{i-1} \equiv \sigma d_{i-1} d_{i} \bmod A^{\prime} \Delta^{\prime}$ with $0<h<A^{\prime} \Delta^{\prime} / d_{i}$,

$$
r_{i} \equiv \frac{d_{i} T}{\Delta_{1}^{\varepsilon_{i}}}-g_{i} \quad \bmod \frac{A^{\prime} \Delta^{\prime}}{d_{i}},
$$

with $0 \leq r_{i} \leq A^{\prime} \Delta^{\prime} / d_{i}$, the first inequality must be strict if $\Delta>0$, and the second inequality must be strict if $\Delta<0$. Here, we recall that $\varepsilon_{i}=\left(1-(-1)^{i}\right) / 2 \in\{0,1\}$ is the parity of $i \in \mathbb{Z}$.

Also define $q_{0}(X)=A X+\lfloor B / A\rfloor-\eta$ and

$$
q_{i}(X)=\frac{2 A(X-K)}{\Delta_{1}^{\varepsilon_{i}}\left(\Delta^{\prime} / d_{i}\right)^{2}}+\left\lfloor\frac{2\left(A^{2} K+B\right)-A \eta-r-G g_{i} \Delta_{1}^{\varepsilon_{i}} \Delta^{\prime} / d_{i}}{A \Delta_{1}^{\varepsilon_{i}}\left(\Delta^{\prime} / d_{i}\right)^{2}}\right\rfloor
$$

for $1 \leq i \leq \kappa$.

The proof of [2, Theorem 3.1] established that $d_{i} \mid \Delta^{\prime}$ for all $i \geq 0$. Since $\Delta^{\prime}$ is coprime to $A^{\prime}$, it follows that $d_{i}=\operatorname{gcd}\left(A^{\prime} \Delta^{\prime} / d_{i-1}, r_{i-1}\right)$, and hence

$$
\operatorname{gcd}\left(\frac{A^{\prime} \Delta^{\prime}}{d_{i-1} d_{i}}, \frac{r_{i-1}}{d_{i}}\right)=1
$$

Thus, the congruence right after (4.2) forces $d_{i-1} \mid h$ and can be rewritten as

$$
\frac{h}{d_{i-1}} \frac{r_{i-1}}{d_{i}} \equiv \sigma \quad \bmod \frac{A^{\prime} \Delta^{\prime}}{d_{i-1} d_{i}}
$$


which always has a solution. Therefore, the quantities presented in formulas (4.1)(4.3) are all well defined.

Theorem 4.1 (Regular continued fraction expansion of $\sqrt{D(X)}$ ). Suppose that $D(X)$ is a Schinzel sleeper. For $X \in \mathbb{Z}$ sufficiently large, the continued fraction expansion of $\sqrt{D(X)}$ is

$$
\sqrt{D(X)}=\left(q_{0}(X) ; \overline{\mathcal{S}_{0}, q_{1}(X), \mathcal{S}_{1}, q_{2}(X), \ldots, \mathcal{S}_{K-1}, q_{\kappa}(X)}\right),
$$

where $\kappa$ is the least positive integer $k$ such that $d_{k}=\Delta^{\prime}$ and $\Delta_{1}^{\varepsilon_{k}}=1$, and $\mathcal{S}_{i}$ is the string of partial quotients in the expansion of $A^{\prime} \Delta^{\prime} / d_{i} r_{i}$, chosen to have odd or even length according to whether $\Delta>0$ or $\Delta<0$, with $\mathcal{S}_{i}$ defined to be the empty string if $r_{i}=0$.

With $d_{i}$ and $r_{i}$ as given in (4.1) and (4.3), respectively, induction easily shows that

$$
\begin{gathered}
\operatorname{gcd}\left(d_{i}, d_{i-1}\right)=1, \\
d_{i-1} Z_{i} \equiv r_{i-1} Z_{i-1} \quad \bmod \Delta^{\prime},
\end{gathered}
$$

for all $i \in \mathbb{N}$, where $Z_{i}$ was defined in (2.3). The following close connection between the quantities $d_{i}$ and $Z_{i}$ is the basis of the proof of Theorem 2.1.

Lemma 4.2. If $i \in \mathbb{N}$, then $d_{i}=\Delta^{\prime}$ if and only if $\Delta^{\prime} \mid Z_{i}$.

Proof. If $\Delta^{\prime} \mid Z_{i}$, then $\Delta^{\prime} \mid d_{i} Z_{i+1}$ by (4.6) (with $i$ replaced by $i+1$ ). By (2.5), $\Delta^{\prime} \mid d_{i}$. Since $d_{i} \mid \Delta^{\prime}$ by the definition of $d_{i}$, we obtain $d_{i}=\Delta^{\prime}$.

Conversely, if $d_{i}=\Delta^{\prime}$, then by the definition of $d_{i}$, we have $\Delta^{\prime} \mid r_{i-1}$. Then (4.6) yields $\Delta^{\prime} \mid d_{i-1} Z_{i}$, and (4.5) forces $\Delta^{\prime} \mid Z_{i}$.

Theorem 4.1 and Lemma 4.2 now immediately yield Theorem 2.1.

From Table 6, we can now deduce an upper bound on $\operatorname{lp}(\sqrt{D(X)})$, the period length of the expansion of $\sqrt{D(X)}$. This is [2, Theorem 4.1] which was stated without proof in that source.

Theorem 4.3 (Period length of the expansion of $\sqrt{D(X)}$ ).

$$
\operatorname{lp}(\sqrt{D(X)}) \leq \begin{cases}3 \Delta^{\prime}\left\lfloor\log _{\varphi}\left(\sqrt{5} A^{\prime} \Delta^{\prime}\right)\right\rfloor & \text { if } \Delta^{\prime} \text { is even, } \\ 2 \Delta^{\prime}\left\lfloor\log _{\varphi}\left(\sqrt{5} A^{\prime} \Delta^{\prime}\right)\right\rfloor & \text { if } \Delta^{\prime} \text { is odd },\end{cases}
$$

where $\log _{\varphi}$ denotes the logarithm to the base $\varphi=(1+\sqrt{5}) / 2$.

Proof. Theorem 4.1 yields

$$
\operatorname{lp}(\sqrt{D(X)})=\sum_{i=0}^{\kappa-1}\left(1+\left|\mathcal{S}_{i}\right|\right) .
$$

By Lamé's theorem (see, for example, Knuth [6, p. 343]),

$$
\left|\mathcal{S}_{i}\right| \leq \log _{\varphi}\left(\sqrt{5} A^{\prime} \Delta^{\prime}\right)-1
$$

for $0 \leq i \leq \kappa-1$, so $\operatorname{lp}(\sqrt{D(X)}) \leq \kappa\left\lfloor\log _{\varphi}\left(\sqrt{5} A^{\prime} \Delta^{\prime}\right)\right\rfloor$. Finally, by Table $6, \kappa \leq 3 \Delta^{\prime}$ if $\Delta^{\prime}$ is even and $\kappa \leq 2 \Delta^{\prime}$ if $\Delta^{\prime}$ is odd. 
TABLE 9. Values of $d_{i}, 0 \leq i \leq 11$, for $\sqrt{30^{2} X^{2}+2 \times 180 X+180}$. Here, $L \equiv X-7$ mod 12 with $-6 \leq L \leq 6$.

\begin{tabular}{rcccccccccccc}
\hline \multicolumn{1}{l}{$L$} & $d_{0}$ & $d_{1}$ & $d_{2}$ & $d_{3}$ & $d_{4}$ & $d_{5}$ & $d_{6}$ & $d_{7}$ & $d_{8}$ & $d_{9}$ & $d_{10}$ & $d_{11}$ \\
\hline-6 & 12 & 1 & 6 & 1 & & & & & & & & \\
-5 & 12 & 1 & 1 & 2 & 3 & 1 & 4 & 1 & 3 & 2 & 1 & 1 \\
-4 & 12 & 1 & 4 & 1 & & & & & & & & \\
-3 & 12 & 1 & 3 & 2 & 3 & 1 & & & & & & \\
-2 & 12 & 1 & 2 & 1 & & & & & & & & \\
-1 & 12 & 1 & 1 & 2 & 3 & 1 & 4 & 1 & 3 & 2 & 1 & 1 \\
$\mathbf{0}$ & $\mathbf{1 2}$ & $\mathbf{1}$ & & & & & & & & & & \\
1 & 12 & 1 & 1 & 2 & 3 & 1 & 4 & 1 & 3 & 2 & 1 & 1 \\
2 & 12 & 1 & 2 & 1 & & & & & & & & \\
3 & 12 & 1 & 3 & 2 & 3 & 1 & & & & & & \\
4 & 12 & 1 & 4 & 1 & & & & & & & & \\
5 & 12 & 1 & 1 & 2 & 3 & 1 & 4 & 1 & 3 & 2 & 1 & 1 \\
6 & 12 & 1 & 6 & 1 & & & & & & & & \\
\hline
\end{tabular}

Note that the bound of Theorem 4.3 is independent of $X$, whence $D(X)$ is a sleeper in the sense of Kaplansky as described in Section 1.

\section{Vertical symmetry}

Theorem 4.1 reveals that the expansion of $\sqrt{D(X)}$ changes according to the congruence class $K$ of $X$ modulo $\Delta^{\prime}$. In fact, the entire expansion of $\sqrt{D(X)}$ depends crucially on the value of $r_{1}$. Recall from Theorem 4.1 that the segments $\mathcal{S}_{i}$, where $i \geq 0$, represent the expansions of the fraction $A^{\prime} \Delta^{\prime} / d_{i} r_{i}$. Suppose that we write the denominators $d_{i} r_{i}$ of these expressions, or even just the values $d_{i}$, in a table, with the rows indexed by $K$ and the columns by $i$. Then this table exhibits a vertical symmetry about a row corresponding to a $K$ value for which $\kappa=2$. This is best illustrated by an example.

Example 5.1. Recall the sleeper $D(X)=30^{2} X^{2}+2 \times 180 X+180$ with $\Delta^{\prime}=12$ from Example 2.4. In Tables 9 and 10, each row corresponds to the expansion of $\sqrt{D(X)}$ with $L \equiv X-7 \bmod 12$, for $-6 \leq L \leq 6$. The entries of each row are the corresponding values of $d_{i}$ and $d_{i} r_{i}$, respectively, for $0 \leq i \leq 11$.

Observe that in Tables 9 and 10, there is a vertical symmetry about the row corresponding to $L=0$ (indicated in boldface), for which $\kappa=2$. Note that there is in fact only one row with $\kappa=2$, which we call the equator of $D(X)$. Referring to the values of $L$ as latitudes, we see that rows of the same absolute latitude yield identical values of $\kappa$. For example, the rows at $L= \pm 1$ both have $\kappa=12$, and the rows at $L= \pm 4$ both have $\kappa=4$. 
TABLE 10. Values of $d_{i} r_{i}, 0 \leq i \leq 11$, for $\sqrt{30^{2} X^{2}+2 \times 180 X+180}$. Here, $L \equiv X-7 \bmod 12$ with $-6 \leq L \leq 6$.

\begin{tabular}{rcccccccccccc}
\hline$L$ & $d_{0} r_{0}$ & $d_{1} r_{1}$ & $d_{2} r_{2}$ & $d_{3} r_{3}$ & $d_{4} r_{4}$ & $d_{5} r_{5}$ & $d_{6} r_{6}$ & $d_{7} r_{7}$ & $d_{8} r_{8}$ & $d_{9} r_{9}$ & $d_{10} r_{10}$ & $d_{11} r_{11}$ \\
\hline-6 & 0 & 6 & 6 & 0 & & & & & & & & \\
-5 & 0 & 11 & 10 & 6 & 9 & 8 & 4 & 3 & 6 & 2 & 1 & 0 \\
-4 & 0 & 4 & 8 & 0 & & & & & & & & \\
-3 & 0 & 9 & 6 & 6 & 3 & 0 & & & & & & \\
-2 & 0 & 2 & 10 & 0 & & & & & & & & \\
-1 & 0 & 7 & 2 & 6 & 9 & 4 & 8 & 3 & 6 & 10 & 5 & 0 \\
$\mathbf{0}$ & $\mathbf{0}$ & $\mathbf{0}$ & & & & & & & & & & \\
1 & 0 & 5 & 10 & 6 & 3 & 8 & 4 & 9 & 6 & 2 & 7 & 0 \\
2 & 0 & 10 & 2 & 0 & & & & & & & & \\
3 & 0 & 3 & 6 & 6 & 9 & 0 & & & & & & \\
4 & 0 & 8 & 4 & 0 & & & & & & & & \\
5 & 0 & 1 & 2 & 6 & 3 & 4 & 8 & 9 & 6 & 10 & 11 & 0 \\
6 & 0 & 6 & 6 & 0 & & & & & & & & \\
\hline
\end{tabular}

Moreover, rows of the same absolute latitude in Table 9 are identical. In Table 10, the entries lying at the intersection of rows of identical absolute latitude with any column sum to $0 \bmod 12$. For example, in Table 9 , the rows with $L= \pm 1$ are identical. In Table 10, the entries lying at the intersection of any of the first four columns with the rows at $L= \pm 4$ add to 0 or 12 .

Last, there are four different patterns, that is, latitudes with the same $\kappa$ value. The four different $\kappa$ values that occur in Tables 9 and 10 are 2, 4, 6 and 12 . Note that 4 , the number of patterns, is a divisor of $\Delta^{\prime}=12$.

We now explore these phenomena in more detail. First, we investigate under what conditions a Schinzel sleeper has an equator, that is, a value of $X \bmod \Delta^{\prime}$ for which $\kappa=2$, and how to find it if it exists. Next, we prove that rows of the same absolute latitude have the same $\kappa$ value. Third, we establish symmetry in any two positions where rows of equal absolute latitude intersect with the same column. Corresponding $d_{i}$ entries are always identical, whilst corresponding $d_{i} r_{i}$ as well as $d_{i} g_{i}$ entries always sum to $0 \bmod \Delta^{\prime}$. Finally, we determine possible values for the number of different patterns (that is, values of $\kappa$ ) and prove that it is always a divisor of $\Delta^{\prime}$.

For the remainder of this section, we assume that $\Delta^{\prime}>1$ as otherwise all patterns collapse into one pattern with either $\kappa=1$ or $\kappa=2$ by Corollary 3.2, making our results trivial. The existence and location of the equator are readily ascertainable.

Theorem 5.2 (Existence and location of equator). A Schinzel sleeper has at most one equator, which exists if and only if

$$
\operatorname{gcd}\left(\frac{2 G}{\Delta_{1} \Delta^{\prime}}, \Delta^{\prime}\right) \mid \frac{2 B^{\prime}}{\Delta_{1} \Delta^{\prime}} .
$$


If it exists, the equator is located at $X \equiv K_{0} \bmod \Delta^{\prime}$ where

$$
A^{\prime 2} \frac{2 G}{\Delta_{1} \Delta^{\prime}} K_{0} \equiv-\frac{2 B^{\prime}}{\Delta_{1} \Delta^{\prime}} \bmod \Delta^{\prime} .
$$

Proof. By Theorems 4.1 and $2.1, \kappa=2$ if and only if $\Delta^{\prime} \mid Z_{2}$. Since $Z_{2}=T / \Delta_{1}$, this is equivalent to $K \equiv K_{0} \bmod \Delta^{\prime}$ with $K_{0}$ as in (5.2). Since $\operatorname{gcd}\left(A^{\prime}, \Delta^{\prime}\right)=1$, the congruence (5.2) has a solution if and only if (5.1) holds, and there is at most one solution $K_{0} \bmod \Delta^{\prime}$.

Assuming that the equator exists, the latitudes are given by $L \equiv K-K_{0} \bmod \Delta^{\prime}$, so the equator is appropriately located at latitude $L=0$. In order to emphasize the dependence on $L$ of some of the quantities defined in Sections 2 and 4, we will henceforth append the subscript $L$, writing $T_{L}, Z_{i, L}, r_{i, L}, d_{i, L}$ and $g_{i, L}$ for $T, Z_{i}, r_{i}, d_{i}$ and $g_{i}$, respectively. Note that specifically

$$
T_{L}=\frac{2\left(A^{2}\left(L+K_{0}\right)+B\right)}{\Delta_{2} \Delta_{4}^{2}}=\frac{2\left(A^{2} L+A^{2} K_{0}+B\right)}{\Delta_{2} \Delta_{4}^{2}} .
$$

To establish vertical symmetry, we first show that any two rows of the same absolute latitude have the same $\kappa$ value. By Theorem 4.1, this amounts to the identity $d_{\kappa, L}=$ $d_{\kappa,-L}=\Delta^{\prime}$. By Theorem 2.1, this is implied by the following lemma.

Lemma 5.3. Suppose that $D(X)$ has an equator. Then

$$
Z_{i, L} \equiv(-1)^{i+1} Z_{i,-L} \bmod \Delta^{\prime}
$$

for all $i \in \mathbb{N}$ and $L \in \mathbb{Z}$.

Proof. The proof proceeds by induction on $i$, keeping $L$ fixed, but arbitrary. Since $Z_{1, L}=1$ for all $L$, this is trivially true for $i=1$. For $i=2$, (5.3) yields

$$
Z_{2, L}+Z_{2,-L}=\frac{T_{L}+T_{-L}}{\Delta_{1}}=2\left(A^{\prime 2} K_{0} \frac{2 G}{\Delta_{1} \Delta^{\prime}}+\frac{2 B^{\prime}}{\Delta_{1} \Delta^{\prime}}\right),
$$

which is a multiple of $\Delta^{\prime}$ by (5.2). Proceeding inductively on $i$, we obtain the desired result.

Next, we prove the symmetry for the entries in rows of equal absolute latitude. Such rows have identical $d_{i}$ values. Moreover, the sum of the $d_{i} r_{i}$ values lying at the intersection of any column with two such rows is divisible by $\Delta^{\prime}$. The latter result in fact also holds for the values $d_{i} g_{i}$.

Theorem 5.4 (Symmetry of opposing row entries). Suppose that $D(X)$ has an equator. Then

$$
\begin{aligned}
d_{i, L} & =d_{i,-L} \\
d_{i+1, L} g_{i+1, L} & \equiv-d_{i+1,-L} g_{i+1,-L} \bmod \Delta^{\prime} \\
d_{i, L} r_{i, L} & \equiv-d_{i,-L} r_{i,-L} \quad \bmod \Delta^{\prime}
\end{aligned}
$$

for $i \geq 0$ and $L \in \mathbb{Z}$. 
Proof. We apply induction on $i$ again, with $L$ fixed. Using Theorem 4.1, we see that $d_{0, K \pm L}=\Delta^{\prime}$ and $d_{1, \pm L}=1$. Also, note that $r_{0, L}$ is independent of $L$. If $r_{0, L} \equiv 0 \bmod A^{\prime} \Delta^{\prime}$, then

$$
d_{1, L} g_{1, L} \equiv-d_{1,-L} g_{1,-L} \equiv 0 \quad \bmod \Delta^{\prime} ;
$$

otherwise, $g_{1, L} \equiv-g_{1,-L} \bmod \Delta^{\prime}$. In both cases, formulas (5.4)-(5.6) hold for $i=0$.

Now since $d_{i, L} \mid \Delta^{\prime}$ for all $L$, we can write

$$
r_{i, L} \equiv-r_{i,-L} \quad \bmod \frac{\Delta^{\prime}}{d_{i, L}}
$$

by the induction hypothesis, so

$$
d_{i+1, L}=\operatorname{gcd}\left(\frac{\Delta^{\prime}}{d_{i, L}}, r_{i, L}\right)=\operatorname{gcd}\left(\frac{\Delta^{\prime}}{d_{i, L}},-r_{i,-L}\right)=d_{i+1,-L} .
$$

This establishes (5.4) for the index $i+1$.

To prove (5.5) for the index $i+1$, by Theorem 4.1, we need to consider the three cases

$$
r_{i, L}=0, \quad r_{i, L}=\frac{A^{\prime} \Delta^{\prime}}{d_{i, L}} \quad r_{i, L} \not \equiv 0 \quad \bmod \frac{A^{\prime} \Delta^{\prime}}{d_{i, L}} ;
$$

similarly for $r_{i,-L}$. If $r_{i, L}=0$, then $g_{i+1, L}=0$, so

$$
d_{i+1, L} g_{i+1, L}=0 .
$$

If $r_{i, L}=A^{\prime} \Delta^{\prime} / d_{i, \pm L}$, then $d_{i+1, L}=\Delta^{\prime} / d_{i, K}$ and $g_{i+1, L}=d_{i, L}$, so

$$
d_{i+1, L} g_{i+1, L}=\Delta^{\prime} \text {. }
$$

Analogous identities hold if $L$ is replaced by $-L$. Note also that $r_{i, L} \in\left\{0, A^{\prime} \Delta^{\prime} / d_{i, L}\right\}$ if and only if $r_{i,-L} \in\left\{0, A^{\prime} \Delta^{\prime} / d_{i,-L}\right\}$ by (5.7). For all four permissible value combinations of this kind, we obtain

$$
d_{i+1, \pm L} g_{i+1, \pm L} \equiv 0 \quad \bmod \Delta^{\prime},
$$

implying (5.5) for the index $i+1$ for the first two cases in (5.8).

For the third case in (5.8), if $r_{i, L} \not \equiv 0 \bmod A \Delta^{\prime} / d_{i, \pm L}$, then it follows from (5.7) that $r_{i,-L} \not \equiv 0 \bmod A \Delta^{\prime} / d_{i, \pm L}$. Thus,

$$
g_{i+1, L} \equiv-g_{i+1,-L} \quad \bmod \Delta^{\prime}
$$

by (5.7) and (4.2), again implying (5.5) for the index $i+1$.

It remains to prove (5.6). By (4.3),

$$
d_{i+1, L} r_{i+1, L} \equiv \frac{d_{i+1, L}^{2} T_{L}}{\Delta_{1}^{\varepsilon_{i+1}}}-d_{i+1, L} g_{i+1, L} \bmod \Delta^{\prime}
$$


and

$$
d_{i+1,-L} r_{i+1, L} \equiv \frac{d_{i+1,-L}^{2} T_{-L}}{\Delta_{1}^{\varepsilon_{i+1}}}-d_{i+1,-L} g_{i+1,-L} \bmod \Delta^{\prime} .
$$

We add these two congruences to obtain (5.6) for the index $i+1$. We have already established that $d_{i+1, L}=d_{i+1,-L}$ and

$$
d_{i+1, L} g_{i+1, L} \equiv d_{i+1, L} g_{i+1, L} \bmod \Delta^{\prime} .
$$

By Lemma 5.3,

$$
\frac{T_{L}+T_{-L}}{\Delta_{1}}=Z_{2, L}+Z_{2,-L} \equiv 0 \quad \bmod \Delta^{\prime},
$$

which proves our claim.

Finally, we investigate how many distinct patterns can occur. Note that $d_{1, L}=1$ for all $L \in \mathbb{Z}$ by Theorem 4.1, so $r_{1, L} \equiv T_{L} / \Delta_{1}-g_{1, L} \bmod A^{\prime} \Delta^{\prime}$ by (4.3). Thus, in light of (1.4) and (5.3), we can write

$$
r_{1, L_{1}}-r_{1, L_{2}} \equiv \frac{T_{L_{1}}-T_{L_{2}}}{\Delta_{1}} \equiv\left(L_{1}-L_{2}\right) A^{\prime 2} \frac{2 G}{\Delta^{\prime} \Delta_{1}} \quad \bmod A^{\prime} \Delta^{\prime}
$$

for all $L_{1}, L_{2} \in \mathbb{Z}$. In particular,

$$
r_{1,0} \equiv r_{1, \Delta^{\prime}} \quad \bmod A^{\prime} \Delta^{\prime}
$$

This means that there can be at most $\Delta^{\prime}$ distinct expansion patterns. Take $\rho$ to be the least value $L$ such that $r_{1,0} \equiv r_{1, L} \bmod A^{\prime} \Delta^{\prime}$; in other words, let $\rho$ be the number of distinct expansion patterns. Then

$$
r_{1,0} \equiv r_{1, \rho} \quad \bmod A^{\prime} \Delta^{\prime}
$$

and if $r_{1, L_{1}} \equiv r_{1, L_{2}} \bmod A^{\prime} \Delta^{\prime}$ with $\left|L_{1}-L_{2}\right|<\rho$, then $L_{1}=L_{2}$. Furthermore,

$$
r_{1, q \rho+L} \equiv r_{1, L} \quad \bmod A^{\prime} \Delta^{\prime} \quad \text { for all } q, L \in \mathbb{Z}
$$

Theorem 5.5 (Maximum number of distinct expansion patterns). We have

$$
\rho \mid \Delta^{\prime} \quad \text { and } \quad \Delta^{\prime} \mid \frac{2 G}{\Delta_{1} \Delta} \rho .
$$

In particular, if $\Delta^{\prime}$ is coprime to $2 G / \Delta_{1} \Delta^{\prime}$, then $\rho=\Delta^{\prime}$.

Proof. The second divisibility follows immediately from (5.9) with $L_{2}=\rho$ and $L_{1}=0$, using the fact that $\operatorname{gcd}\left(A^{\prime}, \Delta^{\prime}\right)=1$. Write $\Delta^{\prime}=q \rho+L$ with $q=\left\lfloor\Delta^{\prime} / \rho\right\rfloor$ and $0 \leq L<\rho$. Then

$$
r_{1,0} \equiv r_{1, \Delta^{\prime}} \equiv r_{1, q \rho+L} \equiv r_{1, L} \quad \bmod A^{\prime} \Delta^{\prime} .
$$

Since $\rho$ is minimal, $L=0$ and hence $\rho \mid \Delta^{\prime}$. 


\section{Generation of all Schinzel sleepers}

Obviously, most triples $(A, B, C)$ of integers with $A>0$ do not satisfy (1.4). It is thus clear that Schinzel sleepers are unusual polynomials that require a very special construction. We now describe a technique that produces all, and nothing but, Schinzel sleepers. The method is particularly useful for generating examples.

Define $\tau$ by means of $\tau=1$ if $\Delta_{1} \Delta^{\prime}$ is odd and $\tau=2$ if $\Delta_{1} \Delta^{\prime}$ is even, so $\Delta_{1} \Delta^{\prime} / \tau \in \mathbb{Z}$. Then (1.4) is equivalent to

$$
\frac{\Delta_{1} \Delta^{\prime}}{\tau} \mid \operatorname{gcd}\left(G, B^{\prime}\right) .
$$

A similar divisibility condition holds for $C$.

Lemma 6.1. $\left(\Delta_{1} \Delta^{\prime 2} / \tau\right) \mid \tau C$.

Proof. We have $\Delta_{1} \Delta^{\prime 2}=\left|B^{\prime 2}-A^{\prime 2} C\right|$. Since $\Delta_{1} \Delta^{\prime 2} \mid \tau^{2} B^{\prime 2}$ by (6.1), we deduce that $\Delta_{1} \Delta^{\prime 2} \mid \tau^{2} A^{\prime 2} C$, and thus

$$
\frac{\Delta_{1} \Delta^{\prime 2}}{\tau} \mid \tau \operatorname{gcd}\left(B^{\prime 2}, A^{\prime 2} C\right)
$$

Now $\operatorname{gcd}\left(B^{\prime 2}, A^{\prime 2} C\right)=\operatorname{gcd}\left(B^{\prime 2}, C\right)$, which is clearly a divisor of $C$. The claim now follows.

Note that Lemma 6.1 implies that $\Delta^{\prime} \mid \tau$ if $C$ is square-free.

Corollary 6.2.

$$
\left(\frac{\Delta^{\prime}}{\operatorname{gcd}\left(\Delta^{\prime}, \tau\right)}\right)^{2} \mid \operatorname{gcd}\left(A^{2}, B, C\right) .
$$

Proof. By (6.1) and Lemma 6.1,

$$
\Delta^{\prime 2} \mid \operatorname{gcd}\left(\tau^{2} G^{2}, \tau^{2} G B^{\prime}, \tau^{2} C\right),
$$

which in turn is a divisor of $\tau^{2} \operatorname{gcd}\left(A^{2}, B, C\right)$.

Corollary 6.2 shows that $\Delta^{\prime} \mid \tau$ under the somewhat weaker assumption that $\operatorname{gcd}\left(A^{2}, B, C\right)$, rather than $C$, is square-free. Of course every nontrivial square factor of $\operatorname{gcd}\left(A^{2}, B, C\right)$ divides $\Delta_{4}$. Hence, $\operatorname{gcd}\left(A^{2}, B, C\right)$ is square-free if $\Delta_{4}=1$. By Corollary $6.2, \operatorname{gcd}\left(A^{2}, B, C\right)$ is not square-free if $\Delta^{\prime}>2$ is even or $\Delta^{\prime}>1$ is odd.

Note that by (6.1) and Lemma 6.1, there exist $l, m, n \in \mathbb{Z}$ with

$$
G=\frac{m \Delta_{1} \Delta^{\prime}}{\tau}, \quad B^{\prime}=\frac{n \Delta_{1} \Delta^{\prime}}{\tau}, \quad C=\frac{l \Delta_{1} \Delta^{\prime 2}}{\tau^{2}} .
$$

Then $B^{\prime 2}-A^{\prime 2} C=\Delta_{1} \Delta^{\prime 2}$ is easily verified to be equivalent to

$$
n^{2} \Delta_{1}-\sigma \tau^{2}=l A^{\prime 2} .
$$

The above identities lead to the following algorithm. 
Algorithm 6.3 (Generation of all Schinzel sleepers).

1. Choose $\sigma \in\{1,-1\}$ and $\Delta_{1} \in \mathbb{N}$ square-free.

2. Choose $\tau \in\{1,2\}$ so that $\tau=2$ whenever $\Delta_{1}$ is even.

3. Choose $n, l \in \mathbb{Z}$ and $A^{\prime} \in \mathbb{N}$ to satisfy (6.3) and in addition $A^{\prime}$ is odd whenever $n$ is even and $\tau=2$.

4. Choose $\Delta^{\prime} \in \mathbb{N}$ so that:

$\Delta^{\prime}$ is even whenever $\Delta_{1}$ is odd and $\tau=2$;

$\Delta^{\prime}$ is odd whenever $\Delta_{1}$ is odd and $\tau=1$; $\operatorname{gcd}\left(\Delta^{\prime}, A^{\prime}\right)=1$.

5. Choose $m \in \mathbb{N}$ and define $G, B^{\prime}, C$ via (6.2).

6. Set $A=G A^{\prime}$ and $B=G B^{\prime}$.

7. Output $D(X)=A^{2} X^{2}+2 B X+C$.

THeORem 6.4. Every output of Algorithm 6.3 is a Schinzel sleeper.

Proof. Note that for all $n \in \mathbb{Z}$, there always exist $l \in \mathbb{Z}$ and $A^{\prime} \in \mathbb{N}$ satisfying (6.3) with $A^{\prime}$ odd whenever $n^{\prime}$ is even and $\tau=2$, as no restriction is placed on $l$.

Furthermore, permissible choices for $\Delta^{\prime}$ exist. We only need to rule out the possibility that $A^{\prime}$ is even and $\Delta^{\prime}$ might need to be chosen even, which would be the case when $\Delta_{1}$ is odd and $\tau=2$. But in that scenario, if $n$ is even, then $A^{\prime}$ is chosen odd according to step 3 , and if $n$ is odd, then $n^{2} \Delta_{1}-\sigma \tau^{2}$ is odd, forcing $A^{\prime}$ to be odd by (6.3).

Let $D(X)=A^{2} X^{2}+2 B X+C$ be the output of Algorithm 6.3 obtained via a choice of parameters $\sigma, \tau, \Delta_{1}, \Delta^{\prime}, A^{\prime}, l, m, n$. Then $\tau \mid \Delta_{1} \Delta^{\prime}$ by construction, so $G$ and $B^{\prime}$ as given in (6.2) are integers. If $\tau=1$ or $\Delta^{\prime}$ is even, then $C$ is also an integer. So suppose that $\tau=2$ and $\Delta^{\prime}$ is odd. Then we need to show that $l$ is even to ensure that $C \in \mathbb{Z}$. By step $4, \Delta_{1}$ is even, so $n^{2} \Delta_{1}$ is also even. By step 3,l$l A^{\prime 2}$ is even. If $n$ is even, then $A^{\prime}$ must be chosen odd, so $l$ is even. If $n$ is odd, then $l A^{\prime 2} \equiv n^{2} \Delta_{1} \equiv 2 \bmod 4$, so $l$ is again even.

As mentioned before, it is easy to verify that (6.3) implies that $B^{\prime 2}-A^{\prime 2} C=$ $\sigma \Delta_{1} \Delta^{\prime 2}$. Thus, by step $6, B^{2}-A^{2} C=\Delta_{1}\left(G \Delta^{\prime}\right)^{2} \neq 0$. The fact that $m$ and $n$ are integers immediately implies (1.4), and hence the Schinzel condition (1.2).

Theorem 6.5. Let $D(X)=A^{2} X^{2}+2 B X+C$ be a Schinzel sleeper. Then there exists a choice of parameters $\sigma, \tau, \Delta_{1}, \Delta^{\prime}, A^{\prime}, l, m, n$ for which Algorithm 6.3 outputs $D(X)$.

Proof. Set $\Delta=B^{2}-A^{2} C, g=\operatorname{gcd}(A, B), a^{\prime}=A / g$, and $b^{\prime}=B / g$. Write $|\Delta|=\delta_{1} \delta_{2}^{2} \delta_{4}^{4}$ with $\delta_{1}, \delta_{2}$ square-free. Then $g=\operatorname{gcd}\left(a^{\prime}, \delta_{2} \delta_{4}^{2}\right)$ by our remarks at the end of Section 1 . Put $\delta^{\prime}=\delta_{2} \delta_{4}^{2} / g \in \mathbb{N}$. Then $\operatorname{gcd}\left(a^{\prime}, \delta^{\prime}\right)=1$.

In steps 1 and 2 of Algorithm 6.3, choose $\sigma=\operatorname{sgn}(\Delta), \Delta_{1}=\delta_{1}, \tau=1$ if $\delta_{1} \delta^{\prime}$ is odd, and $\tau=2$ if $\delta_{1} \delta^{\prime}$ is even. Then $\tau \mid \delta_{1} \delta^{\prime}$.

Next, choose $A^{\prime}=a^{\prime}, n=\tau b^{\prime} / \delta_{1} \delta^{\prime}$, and $l=\tau^{2} C / \delta_{1} \delta^{\prime 2}$ in step 3. Then $A^{\prime} \in \mathbb{N}$ by definition of $g, n \in \mathbb{Z}$ by (6.1), and $l \in \mathbb{Z}$ by Lemma 6.1. Furthermore, $1=\operatorname{gcd}\left(a^{\prime}, b^{\prime}\right)=$ $\operatorname{gcd}\left(A^{\prime}, n \delta_{1} \delta^{\prime} / \tau\right)$, so if $n$ is even, then $A^{\prime}$ is odd. It is also easy to verify that (6.3) holds. 
TABle 11. Generating $D(X)=30^{2} X^{2}+2 \times 180 X+180$ and $D(X)=119^{2} X^{2}+2 \times 1666 X+98$ via Algorithm 6.3.

\begin{tabular}{lll}
\hline Step & $D(X)=30^{2} X^{2}+2 \times 180 X+180$ & $D(X)=119^{2} X^{2}+2 \times 1666 X+98$ \\
\hline 1 & Take $\sigma=-1$ and $\Delta_{1}=1$ & Take $\sigma=1$ and $\Delta_{1}=2$ \\
2 & Take $\tau=2$ & Take $\tau=2$ \\
3 & Set $n=1, l=5$ and $A^{\prime}=1$ & Set $n=2, l=4$ and $A^{\prime}=1$ \\
4 & Take $\Delta^{\prime}=12$ & Take $\Delta^{\prime}=7$ \\
5 & Take $m=5$, & Take $m=17$, \\
& then $G=30, B^{\prime}=6, C=180$ & then $G=119, B^{\prime}=14, C=98$ \\
6 & $A=30$ and $B=180$ & $A=119$ and $B=1666$ \\
\hline
\end{tabular}

In step 4 , choose $\Delta^{\prime}=\delta^{\prime}$. Then $\operatorname{gcd}\left(A^{\prime}, \Delta^{\prime}\right)=\operatorname{gcd}\left(a^{\prime}, \delta^{\prime}\right)=1$. Moreover, by definition of $\tau$, we see that $\Delta^{\prime}$ is even if $\Delta_{1}$ is odd and $\tau=2$, and $\Delta^{\prime}$ is odd if $\Delta_{1}$ is odd and $\tau=1$.

Last, in step 5, chose $m=\tau g / \delta_{1} \delta^{\prime}$. Then $m \in \mathbb{N}$ by (6.1). Finally, (6.2) and step 6 yield $G=g, B^{\prime}=b^{\prime}, l \Delta_{1} \Delta^{\prime 2} / \tau^{2}=C, A^{\prime} G=a^{\prime} g=A$, and $B^{\prime} G=b^{\prime} g=B$. Thus, Algorithm 6.3 outputs $D(X)$.

Theorems 6.4 and 6.5 show that Algorithm 6.3 does in fact generate exactly those quadratic polynomials that are Schinzel sleepers.

ExAmple 6.6. The two Schinzel sleepers from Examples 2.4 and 2.5, namely $D(X)=$ $30^{2} X^{2}+2 \times 180 X+180$ and $D(X)=119^{2} X^{2}+2 \times 1666 X+98$, can be generated by Algorithm 6.3 as described in Table 11.

We conclude with a few remarks.

Remark 6.7. In order to avoid the case $\operatorname{gcd}\left(A^{2}, B, C\right)$ square-free which was considered in [14], by Corollary 6.2, it suffices to choose $\Delta^{\prime}>2$ even or $\Delta^{\prime}>1$ odd in step 4 of Algorithm 6.3.

On the other hand, if $\operatorname{gcd}\left(A^{2}, B, C\right)$ is desired to be square-free, it is obviously necessary to choose $\Delta^{\prime} \mid \tau$ by Corollary 6.2. Since $\Delta_{2} \Delta_{4}^{2}=G \Delta^{\prime}$, we have $\Delta_{2} \Delta_{4}^{2}=m \Delta_{1} / \tau$ if $\Delta^{\prime}=1$ and $\Delta_{2} \Delta_{4}^{2}=2 m \Delta_{1}$ if $\Delta^{\prime}=2$. Hence, to ensure that $\Delta_{4}=1$, it suffices to choose $m$ in step 5 square-free and coprime to $\Delta_{1} \Delta^{\prime} / \tau$.

Remark 6.8. By [14, Theorem 2.2], if $C \leq 0$ or $C$ is a perfect square, then $D(X)$ is of Richaud-Degert type, that is, $D(X)=R(X)^{2}+S(X)$ with $R(X), S(X)$ linear in $X$ and $S(X) \mid 4 R(X)$. The expansions for these types of surd are well known. To avoid this scenario, select $n>\tau / \sqrt{\Delta_{1}}$ in step 3 of Algorithm 6.3 to ensure that $C>0$, and choose $n l \neq 0$ whenever $\Delta_{1}=1$ to guarantee that $C$ is not a square. 
Remark 6.9. Assume now that $C>0$ is not a perfect square. Then it is well known that if $\left|B^{\prime}\right| / A^{\prime}$ is a convergent in the expansion of $\sqrt{C}$, then

$$
\left|\frac{\left|B^{\prime}\right|}{A^{\prime}}-\sqrt{C}\right|<\frac{1}{A^{\prime 2}},
$$

or equivalently,

$$
\Delta^{\prime}<\frac{1}{\tau}\left(\frac{|n|}{A^{\prime}}+\sqrt{\frac{l}{\Delta_{1}}}\right)=\frac{\tau}{A^{\prime}\left|n \Delta_{1}-A^{\prime} \sqrt{l \Delta_{1}}\right|} .
$$

Thus, if $\Delta^{\prime}$ is chosen sufficiently large in step 4 of Algorithm 6.3, then $\left|B^{\prime}\right| / A^{\prime}$ is a not convergent in the expansion of $\sqrt{C}$.

On the other hand, if $\sqrt{C}>\Delta_{1} \Delta^{\prime 2}$, then $\left|B^{\prime 2}-A^{\prime 2} C\right|=\Delta_{1} \Delta^{\prime 2}<\sqrt{C}$ implies that

$$
\left|\frac{\left|B^{\prime}\right|}{A^{\prime}}-\sqrt{C}\right|<\frac{1}{A^{\prime}\left(\frac{\left|B^{\prime}\right|}{\sqrt{C}}+A^{\prime}\right)}<\frac{1}{2\left(A^{\prime}\right)^{2}},
$$

in which case $\left|B^{\prime}\right| / A^{\prime}$ is a convergent in the expansion of $\sqrt{C}$. One can force this by choosing $\Delta^{\prime}$ sufficiently small so that $\Delta^{\prime 2}<l / \tau^{2} \Delta_{1}$ in Algorithm 6.3.

It was noted in [14, p. 26] that replacing $X$ by $Y=X+X_{0}$ with $X_{0}>\Delta_{1} \Delta^{\prime 2} / A$ results in a Schinzel sleeper $\tilde{D}(Y)=A^{2} Y^{2}+2 \tilde{B} Y+\tilde{C}$ with the properties $\tilde{B}^{2}-A^{2} \tilde{C}=$ $\Delta, \operatorname{gcd}\left(A^{2}, \tilde{B}\right)=G, \tilde{C}>0$, and $\sqrt{\tilde{C}}>\Delta_{1} \Delta^{\prime 2}$. Then $\tilde{D}(Y)$ will have the same expansion patterns as $D(X)$, but the corresponding $K$ and $L$ values are shifted by $X_{0}$. See also [14, Theorems 4.3 and 4.4] for the connection between $\sqrt{C}$ and $\sqrt{D(X)}$ in the case when $\operatorname{gcd}\left(A^{2}, 2 B, C\right)$ is square-free.

\section{Conclusion}

Let $D(X)=A^{2} X^{2}+2 B X+C$ be a quadratic polynomial such that $A \in \mathbb{N}$ and $B, C, X \in \mathbb{Z}$. In general, one would expect the period length of the continued fraction expansion of $\sqrt{D(X)}$ to fluctuate wildly as $X$ varies. The work of Schinzel [10] established that this is indeed the case unless $D(X)$ is a Schinzel sleeper. Specifically, the period length of the expansion of $\sqrt{D(X)}$ is bounded as $X$ grows if and only if $\Delta=B^{2}-A^{2} C$ is a divisor of $4 \operatorname{gcd}\left(A^{2}, B\right)^{2}$. Moreover, in this case, it is possible to write down the entire expansion of $\sqrt{D(X)}$ for any $X$ as described in Theorem 4.1. Ignoring the first term $\lfloor\sqrt{D(X)}\rfloor$, this expansion is comprised of a number of segments. Each such segment consists of the partial quotients of the expansion of a certain rational number, followed by a linear polynomial in $X$.

Arguably the most important quantity associated with a Schinzel sleeper $D(X)$ is the value $\Delta^{\prime}$, whose square is defined to be the square kernel of $|\Delta| / \operatorname{gcd}(A, B)^{2}$. Much of the behaviour of the expansion of $\sqrt{D(X)}$ is governed by $\Delta^{\prime}$. Note that $\Delta^{\prime}$ is trivial, that is, equal to 1 or 2 , if $\operatorname{gcd}\left(A^{2}, B, C\right)$ is square-free, which is always the case if $\Delta$ is free of fourth powers. The number $\kappa$ of segments in the expansion of $\sqrt{D(X)}$, or $\kappa / 2$ if $\kappa$ is even, is exactly the rank of apparition of $\Delta^{\prime}$, or of a certain factor of $\Delta^{\prime}$ if $\kappa$ is 
even, with respect to a particular Lucas sequence that depends only on $A, B, C$, and $X$ mod $\Delta^{\prime}$. It is thus clear that $\kappa$ can take on at most $\Delta^{\prime}$ different values as $X$ varies, and the number of different $\kappa$ values (patterns) is in fact a divisor of $\Delta^{\prime}$. While it is possible to provide explicit upper bounds on the period length of the expansion of $\sqrt{D(X)}$ that depend only on $A, B, C$, it seems difficult in general to determine the exact value of $\kappa$. However, in certain cases, such as small period lengths or certain prime values of $\Delta^{\prime}$, it is possible to a priori determine $\kappa$ without actually having to compute the expansion of $\sqrt{D(X)}$.

In addition to the usual horizontal symmetry exhibited by the continued fraction expansion of any quadratic surd, Schinzel sleepers have additional vertical symmetries as $X$ varies across the different congruence classes of $X$ modulo $\Delta^{\prime}$. For different such congruence classes, the expansions of $\sqrt{D\left(X \bmod \Delta^{\prime}\right)}$ exhibit patterns that are symmetric with respect to a congruence class for which $\kappa=2$, provided such a class exists. The existence of this equator, and (in the affirmative) its location are easily established. A further investigation into potential connections between the values of $\kappa$ and the corresponding latitudes is currently in progress.

The Cohen-Lenstra heuristics [3] predict that roughly 75 percent of all real quadratic fields have class number 1 , and hence a very large regulator. This in turn translates into a large period length. Large here means that the regulator is about the square root of the discriminant of the field. Schinzel sleepers represent an extreme exception to the Cohen-Lenstra heuristics, with a period length that is not only small, but bounded. Due to the restrictiveness of the Schinzel condition (1.2), they need to be constructed with care. A procedure for generating each and every Schinzel sleeper was given as Algorithm 6.3 in Section 6. This method can also ensure certain conditions on the Schinzel sleepers it generates. For example, it can force $\operatorname{gcd}\left(A^{2}, B, C\right)$ to contain a square factor (or $|\Delta|$ to contain a fourth power), or it can guarantee that the quotient $A /|B|$ occurs or does not occur as a convergent in the expansion of $\sqrt{C}$ when $C>0$ and $\Delta>0$.

Schinzel sleepers represent by no means the only parameterized family of integers that give rise to quadratic surds with small period lengths, and an extensive body of literature exists on this topic. For a detailed history and thorough overview on the subject, the reader is referred to the two doctoral dissertations [1, 7]. In this context, Kaplansky coined the rather facetious nomenclature sleepers, creepers, and leapers; we already encountered the first of these three terms earlier. In essence, sleepers have bounded period length, creepers have slowly growing period length, and leapers are generic discriminants whose period length grows exponentially. Subsequently, van der Poorten added beepers, so named in honour of the beer he won in a mathematical challenge posed to him by Williams [13]. Beepers have unbounded period length, but their regulator grows logarithmically in the discriminant, whereas all known creepers are polynomially parameterized so that the period length grows linearly and the regulator quadratically in the degree.

Schinzel $[11,12]$ completely settled the question of when exactly a polynomial of arbitrary degree is a sleeper. The complete expansion arising from a Schinzel sleeper, 
as described in Theorem 4.1, was first described in [2]; see also [1] for a detailed investigation of Schinzel sleepers. Creepers have enjoyed considerable study; see, for example, [15] and the literature listed in that source. A special case of creepers are the kreepers $D_{n}(X)=A^{2} X^{2 n}+B X^{n}+C^{2}$, so named in honour of Kaplansky, which were fully characterized in $[7,8]$. They are creepers whose period length grows linearly in $n$ and for which there exists a reduced principal ideal in the maximal order of the quadratic field $\mathbb{Q}\left(\sqrt{D_{n}(X)}\right)$ whose norm is a fixed power of $X$ that is independent of $n$.

It is an ostensibly difficult open question whether there exist parameterized families with slowly growing period lengths (that is, are not leapers) other than the three types named by Kaplansky and van der Poorten.

\title{
References
}

[1] K. H. F. Cheng, 'Some results concerning periodic continued fractions', Doctoral Dissertation, University of Calgary, Canada, 2003.

[2] K. H. F. Cheng and H. C. Williams, 'Some results concerning certain periodic continued fractions', Acta Arith. 117 (2005), 247-264.

[3] H. Cohen and H. W. Lenstra Jr, 'Heuristics on class groups of number fields', in: Number Theory (Noordwijkerhout 1983), Lecture Notes in Mathematics, 1068 (Springer, Berlin, 1984), pp. 33-62.

[4] M. J. Jacobson Jr and H. C. Williams, Solving the Pell Equation, CMS Books in Mathematics (Springer, New York, 2009).

[5] I. Kaplansky, 'Letter to R. A. Mollin, H. C. Williams and K. S. Williams', 1998.

[6] D. Knuth, The Art of Computer Programming, vol. 2. Seminumerical algorithms, 2nd edn (Addison-Wesley, Reading, MA, 1981).

[7] R. D. Patterson, 'Creepers - real quadratic fields with large class number', Doctoral Dissertation, Macquarie University, Sydney, 2003.

[8] R. D. Patterson, A. J. van der Poorten and H. C. Williams, 'Characterization of a generalized Shanks sequence', Pacific J. Math. 230 (2007), 185-215.

[9] P. Ribenboim, The Book of Prime Number Records (Springer, New York, 1988).

[10] A. Schinzel, 'On some problems of the arithmetical theory of continued fractions', Acta Arith. 6 (1961), 393-413.

[11] A. Schinzel, 'On some problems of the arithmetical theory of continued fractions II', Acta Arith. 7 (1962), 187-298.

[12] A. Schinzel, 'Corrigendum to "On some problems of the arithmetical theory of continued fractions II", Acta Arith. 47 (1986), 295.

[13] A. J. van der Poorten, 'Beer and continued fractions with periodic periods', in: Number Theory (Ottawa, ON, 1996), CRM Proceedings and Lecture Notes, 19 (American Mathematical Society, Providence, RI, 1999), pp. 309-314.

[14] A. J. van der Poorten and H. C. Williams, 'On certain continued fraction expansions of fixed period length', Acta Arith. 89 (1999), 23-35.

[15] H. C. Williams, 'Some generalizations of the $S_{n}$ sequence of Shanks', Acta Arith. 69 (1995), 199-215.

[16] H. C. Williams, Édouard Lucas and Primality Testing, Canadian Mathematical Society Series of Monographs and Advanced Texts, 22 (Wiley, New York, 1998).

\author{
K. H. F. CHENG, Department of Mathematics and Information Technology, \\ The Hong Kong Institute of Education, 10 Lo Ping Road, \\ Tai Po, New Territories, Hong Kong \\ e-mail: khfcheng@ied.edu.hk
}


R. K. GUY, Department of Mathematics and Statistics, University of Calgary, 2500 University Drive NW, Calgary, Alberta, Canada T2N 1N4 e-mail: rkg@cpsc.ucalgary.ca

R. SCHEIDLER, Department of Mathematics and Statistics, University of Calgary, 2500 University Drive NW, Calgary, Alberta, Canada T2N 1N4 e-mail: rscheidl@ucalgary.ca

H. C. WILLIAMS, Department of Mathematics and Statistics, University of Calgary, 2500 University Drive NW, Calgary, Alberta, Canada T2N 1N4

e-mail: hwilliam@ucalgary.ca 